\title{
Periosteal Osteosarcoma of the Mandible: Case Report and Review of the Literature
}

\author{
Guo Dong Wang, M.D., Yun Fu Zhao, M.D.., Yuan Liu, M.D., Lei Jiang, M.D., and Xiao Zhong \\ Jiang, M.D.
}

Abstract

Purpose-Periosteal osteosarcoma is an intermediate-grade chondroblastic osteosarcoma, accounting for less than $2 \%$ of all osteosarcomas and it occurs extremely rarely in the jaws. We present a rare case of mandibular periosteal osteosarcoma.

Patients and Methods-A 39-year-old man presented with a four months history of a firm swelling of the mental region. The clinical, radiologic, and histologic findings pointed to a diagnosis of periosteal osteosarcoma.

Results-The patient underwent a partial mandibulectomy. After ten months the lesion recurred locally. We removed the tumor together with the residual right mandible, and the patient remained without evidences of local recurrence after 22 months.

Conclusions-Periosteal osteosarcoma may give rise to local recurrence and distant metatasis. It is important to differentiate the periosteal osteosarcoma from other types. Accurate diagnosis requires correlation of clinical, radiographic, and histologic features, and wide surgical margins are mandatory for successful therapy.

\section{Keywords}

periosteal osteosarcoma; juxtacortical osteosarcoma; mandible; recurrence; surface osteosarcoma

Osteosarcoma (OS) is the most common primary malignancy of bone, with a reported incidence of 1:100,000 per year. 1 These tumors typically originate in the extremities and the pelvis, with only $2.7-6.6 \%$ of OSs were located in the jaws.2.3

Although conventional OS arises intramedullary, some OSs occur on the outer surface of bone, this is called surface or juxtacortical OS and exhibits distinct radiographic, histologic features and biological behavior that is obviously different from conventional OS. OSs arising on the surface of bone can be classified into three types according to the nature of the tumor: parosteal, periosteal, and high-grade surface OS.4 Parosteal OS is a low-grade OS, with an incidence of about $4 \%$ of all OSs. Periosteal OS is an intermediate-grade chondroblastic OS, accounting for less than $2 \%$ of all OSs. High-grade surface OS is the most rare subtype, accounting for less than $1 \%$. Periosteal type tends to have a better

\footnotetext{
(C) 2010 The American Association of Oral and Maxillofacial Surgeons. Published by Elsevier Inc. All rights reserved.

"Correspondence to: Yun Fu Zhao, Address: Department of Stomatology, Changzheng Hospital, Second Military Medical University, 415 Fengyang Rd, Shanghai, 200003, P. R. China. Phone number: 86-021-81885941, Fax number: 86-021-63528552, zhaoyf1818@126.com.

Publisher's Disclaimer: This is a PDF file of an unedited manuscript that has been accepted for publication. As a service to our customers we are providing this early version of the manuscript. The manuscript will undergo copyediting, typesetting, and review of the resulting proof before it is published in its final citable form. Please note that during the production process errors may be discovered which could affect the content, and all legal disclaimers that apply to the journal pertain.
} 
prognosis than that of conventional OS or high-grade surface OS, but poorer than that of parosteal OS and it is capable of local recurrence and distant metastasis.5

Periosteal OS occurs extremely rarely in the jaws. To our knowledge, including this report, only ten cases have been reported. We report a case of periosteal OS located at the mental region of the mandible and review the literature with respect to clinical, radiological and histopathological criteria, we also discuss the differential diagnosis.

\section{Report of a case}

A 39-year-old man was admitted because of swelling of the mental region and numbness on the overlying skin. His symptoms started four months earlier when he initially noticed a gradually enlarging mass in the mental region. Since his lower incisors had been mobile for several months, he consulted a countryside dentist, who recommended removal of his mobile teeth. Consequently, the lower central incisors and lower right lateral incisor were removed by the dentist but the mass failed to resolve. During the next 4 months, the lesion continued to increase in size gradually. The patient was subsequently referred to our hospital for further management. Before seeking treatment, he developed numbness of the mental region. His past history and family history were not contributory, and no apparent history of trauma around the mental region was noted.

On examination, an approximately $4 \times 5 \mathrm{~cm}$ mass was found to overlay the mental region (Figure 1). The mass was rubbery, nontender, and fixed on palpation. There was no palpable cervical lymphadenopathy. Intraoral examination revealed mandibular vestibule and the mouth floor swelling with expansion extending from the lower right first premolar to the lower left lateral incisor. The swelling was covered with intact, normal appearing mucosa. The lower incisors extraction sockets had healed. The other teeth were not mobile. Laboratory data including alkaline phosphatase level were within normal limits. Because osteogenic neoplasms were higher on the differential diagnostic list, a mandible CT scan was ordered.

CT scan of the mandible revealed a well circumscribed homogeneous and radiolucent mass measuring $30 \mathrm{~mm} \times 46 \mathrm{~mm}$ in size, with non-homogeneous contrast enhancement. The tumor was found to encircle the cortex of the bone partially. There was no evidence of cortical destruction or medullary involvement (Figure 2).

On the basis of these findings, the patient was counseled regarding the need for an incisional biopsy. At the time of surgery, after elevation of a mucoperiosteal flap, the lesion was found to be closely attached to the cortical plate of the mandible, but the cortex had remained intact. Histopathological examination of an incisional biopsy specimen revealed that the tumor consisted exclusively of lobules of cartilaginous-appearing tissue with small foci of osteoid formation. The chondroid matrix component was composed of poorly differentiated, anaplastic chondroblastic cells (Figure 3A). In addition, mitotic figures were readily seen among the chondrocytes and osteocytes (Figure 3B). Immunohistochemically, the tumor showed diffuse reactivity with antibodies against vimentin and focal reactivity with antibodies against the S-100 protein in the chondroid containing fields and the spindle cells (Figure 3C). On the basis of clinical, radiological, histopathological features, a diagnosis of periosteal OS of the mandible was established. After the diagnosis of periosteal OS had been made, the patient underwent a partial mandibulectomy from the lower right second premolar to the lower left canine with the overlying skin and mucosa and immediate reconstruction with a titanium plate and bilobed radial forearm free flap. No residual tumor was identified. After ten months the lesion recurred locally, which was located at the lingual bone cortex surface of the residual mandible on the right side (Figure 4A). We removed the tumor together with the residue of the right mandible (Figure 4B). The resected primary and 
recurrent lesions demonstrated histopathologic features identical to those seen in the initial biopsy specimen. At no time was radiation or chemotherapy given. 22 months after the second surgery, he was without evidence of disease recurrence or distant metastasis.

\section{Discussion}

Periosteal OS is an uncommon neoplasm that comprises less than $2 \%$ of all OSs 4 and is rarely located in the jaws. To date, including this report, only 10 cases of periosteal OS of the jaws have now been reported in the English-language literature. $5^{-} 12$ There were 5 males and 5 females with an age range from 16 to 65 years and a median of 33 years. Our case was in this age range. Three tumors arose in the maxilla and seven in the mandible. Our case arose in the mandible. All patients underwent surgery to excise the tumor. The median follow-up is 30 months (range $8-72$ months). With the exception of only 2 (this case included) of the 10 cases reported, there have been no recurrences of tumor. In the first case, described by Piatelli and Favia,5 tumor recurred locally 18 months later. The patient underwent a radical right maxillectomy followed by several cycles of adjuvant chemotherapy. Lung metastases appeared, and the patient died 5 years after the first diagnosis.

The definition of periosteal OS is very precise. Accurate diagnosis requires correlation of clinical, radiographic, and histologic features. Importantly it is essential to exclude intramedullary OS with periosteal extension, high-grade surface OS and parosteal OS from this group. The clinicopathologic characteristics of periosteal OS arising in the jaws include the following: (1) peak occurrence is over 30 years of age, the median age of periosteal OS of the jaws is about 15 years later than that in the long bone counterparts;13 (2) the tumor is most often lobulated and well defined; (3) radiologically, periosteal OS tends to be more radiolucent than the other types of OS since it is predominantly cartilaginous, 2,6 the cortex is intact and sometimes thickened, and there may be minimal tumor invasion into the cortex, without medullary involvement;12 (4) the tumor is predominantly composed of poorly differentiated malignant cartilaginous tissue;11, 12 (5) the tumor may give rise to local recurrence and distant metatasis;5 (6) prognosis in periosteal OS seems to be excellent with surgical treatment 2 only and is considerably better than that of conventional OS or highgrade surface OS, but poorer than that of parosteal OS.6 The clinical presentation of the parosteal variant 14, 15 of OS is as an expansile lesion on the involved bone, with a nonlobular or lobular outer surface, and a potential for overlying mucosal ulceration. Radiologically, parosteal OS appears as a hyperdense, homogenous mass similar to compact bone attached to the cortex by a thin layer of hypodense tissue. Histologically, this tumor is well differentiated and characterized by a spindle-cell fibroblastic-like proliferation containing well-developed bony trabeculae. Our patient's tumor was dominated by aberrant chondroid with a lesser component of osteoid. The CT images of this case revealed that the tumor was a homogeneous and radiolucent mass with no evidence of involvement of the marrow cavity. High-grade surface OS16 is characterized by a rapid-growing sarcoma arising from periosteal tissue. Radiologically, the tumor appears as dense to moderate mineralization with a fluffy, immature appearance. Histology reveals a highly pleomorphic, predominantly osteoblastic, lesion that lacks the cartilaginous lobules seen in periosteal OS. The criteria for a diagnosis of high grade surface OS are that the lesion had arisen on the surface of bone, that histologic findings of the tumor are identical to those of conventional intramedullary OS and that, even if medullary involvement is present, most of the tumor is on the surface of bone.17, 18 Conventional OS typically is of a higher histologic grade than its periosteal counterpart. Radiographs of most conventional OSs of the jaws show a unicentric bone destructive lesion with indefinite margins. The radiological appearance usually will be sclerotic, lytic or mixed, in which areas of calcification and of lysis are intermingled. Histologically, conventional OS is characterized by tumor osteoid and/or 
osseous tissue arising directly from a sarcomatous stroma without passing through a cartilaginous phase. The stromal cells may be predominantly osteoblastic, chondroblastic or fibroblastic.19 The key differences in four types of OSs are summarized in Table 1. In our patient's case, an intramedullary OS with periosteal extension and a high-grade surface OS were ruled out because the tumor originated and initially proliferated outward from the osseous surface and without intramedullary extension.

Revell et al13 described the tumor was found to encircle between 5 and $100 \%$ of the cortex of the bone in their series of periosteal OS arising in the long bone, similar to what was observed in our case. This is the first documented case of a periosteal OS of the mandible with partial cortex encirclement. The overlying skin numbness in the present case could be the result of the right mental nerve involved. The local recurrence in our patient was found after ten months later. We performed a radical en bloc resection of the right mandible. At the 32 month follow-up appointment after the first diagnosis, there was no evidence of recurrent disease or distant metastasis.

In the jaws, OS has different clinical and biologic characteristics than its counterpart in the long bones. $1^{-} 3,20,21$ OS of the jaws tends to occur at an older mean age, and the prognosis for jaw OS is better than that of OS arising in other sites. The more favorable prognosis may be due to lower mitotic activity of tumor cells and because cellular anaplasia is found less often in OS of the jaws. In addition, jaw OSs have less of a tendency to metastasize than OSs of the long bones. However, the survival rate of periosteal OS of the jaws cannot be accurately assessed because of the limited number of cases and lack of longterm follow-up. The most effective therapy for periosteal OS of the jaws is wide surgical excision of either the mandible or the maxilla with a tumor-free margin but the role of chemotherapy in this condition is still debatable. $1,5^{-} 13$

The clinical, radiologic, and microscopic features of our case fulfill the criteria proposed for periosteal OS. Periosteal OS may give rise to local recurrence and distant metatasis. It is important to differentiate the periosteal OS from other types. Accurate diagnosis requires correlation of clinical, radiographic, and histologic features, and wide surgical margins are mandatory for successful therapy.

\section{Acknowledgments}

The authors gratefully acknowledge Dr. Yu Li Li, at the Department of Pathology, Changzhen hospital, The Second Military Medical University, Shanghai, for kindly reviewing the histopathology slides and confirming the diagnosis.

\section{References}

1. Sturgis EM, Potter BO. Sarcomas of the head and neck region. Curr Opin Oncol. 2003; 15:239. [PubMed: 12778019]

2. Unni, KK. Osteosarcoma. In: Unni, KK., editor. Dahlin's Bone Tumors: General aspects and data on 11,087 cases. 5th ed.. Philadelphia: Lippincott-Raven; 1996. p. 143-183.

3. Bertoni F, Dallera P, Bacchini P, et al. The Istituto Rizzoli-Beretta experience with osteosarcoma of the jaw. Cancer. 1991; 68:1555. [PubMed: 1893357]

4. Unni, KK.; Knuutila, S. Fletchcher, CDM.; Unni, KK.; Mertens, F., editors. World Health Organization classification of tumors: pathology and genetics of tumors of soft tissue and bone. Lyon, France: IARC Press; 2002. Parosteal osteosarcoma. p. 279-285.

5. Piattelli A, Favia GF. Periosteal osteosarcoma of the jaws: Report of 2 cases. J Periodontol. 2000; 71:325. [PubMed: 10711625]

6. Zarbo RJ, Regezi JA, Baker SR. Periosteal osteogenic sarcoma of the mandible. Oral Surg Oral Med Oral Pathol. 1984; 57:643. [PubMed: 6588346] 
7. Millar BG, Browne RM, Flood TR. Juxtacortical osteosarcoma of the jaws. Br J Oral Maxillofac Surg. 1990; 28:73. [PubMed: 2186801]

8. Patterson A, Greer RO Jr, Howard D. Periosteal osteosarcoma of the maxilla: a case report and review of literature. J Oral Maxillofac Surg. 1990; 48:522. [PubMed: 2184205]

9. Sorensen DM, Gokden M, El-Naggar A, et al. Periosteal osteosarcoma (PO) of the mandible. Arch Otolaryngol Head Neck Surg. 2000; 126:550. [PubMed: 10772315]

10. Koyama J, Ito J, Hayashi T. Periosteal osteosarcoma of the mandible. Dentomaxillofac Radiol. 2002; 31:63. [PubMed: 11803391]

11. Minic AJ. Periosteal osteosarcoma of the mandible. Int J Oral Maxillofac Surg. 1995; 24:226. [PubMed: 7594757]

12. Yoon JH, Yook JI, Kim HJ, et al. Periosteal osteosarcoma of the mandible. J Oral Maxillofac Surg. 2005; 63:699. [PubMed: 15883947]

13. Revell MP, Deshmukh N, Grimer RJ, et al. Periosteal osteosarcoma: a review of 17 cases with mean follow-up of 52 months. Sarcoma. 2002; 6:123. [PubMed: 18521348]

14. Hewitt KM, Ellis G, Wiggins R, et al. Parosteal osteosarcoma: case report and review of the literature. Head Neck. 2008; 30:122. [PubMed: 17615569]

15. Balwani SR, Tupkari JV, Barpande SR. Parosteal osteosarcoma of the mandible. J Oral Maxillofac Pathol. 2006; 10:10.

16. Wold LE, Unni KK, Beabout JW, Pritchard DJ. High grade surface osteosarcomas. Am J Surg Pathol. 1984; 8:181. [PubMed: 6584042]

17. Okada K, Unni KK, Swee RG, Sim FH. High grade surface osteosarcoma. A clinicopathologic study of 46 cases. Cancer. 1999; 85:1044. [PubMed: 10091787]

18. Staals EL, Bacchini P, Bertoni F. High-grade surface osteosarcoma A review of 25 cases from the Rizzoli institute. Cancer. 2008; 112:1952.

19. Garrington GE, Scofield HH, Cornyn J, Hooker SP. Osteosarcoma of the jaws. Analysis of 56 cases. Cancer. 1967; 203:377. [PubMed: 5228026]

20. Forteza G, Colmenero B, López-Barea F. Osteogenic sarcoma of the maxilla and mandible. Oral Surg Oral Med Oral Pathol. 1986; 62:179. [PubMed: 3462614]

21. Regezi JA, Zarbo RJ, McClatchey KD, Courtney RM, Crissman JD. Osteosarcomas and chondrosarcomas of the jaws: immunohistochemical correlations. Oral Surg Oral Med Oral Pathol. 1987; 64:302. [PubMed: 2443891] 


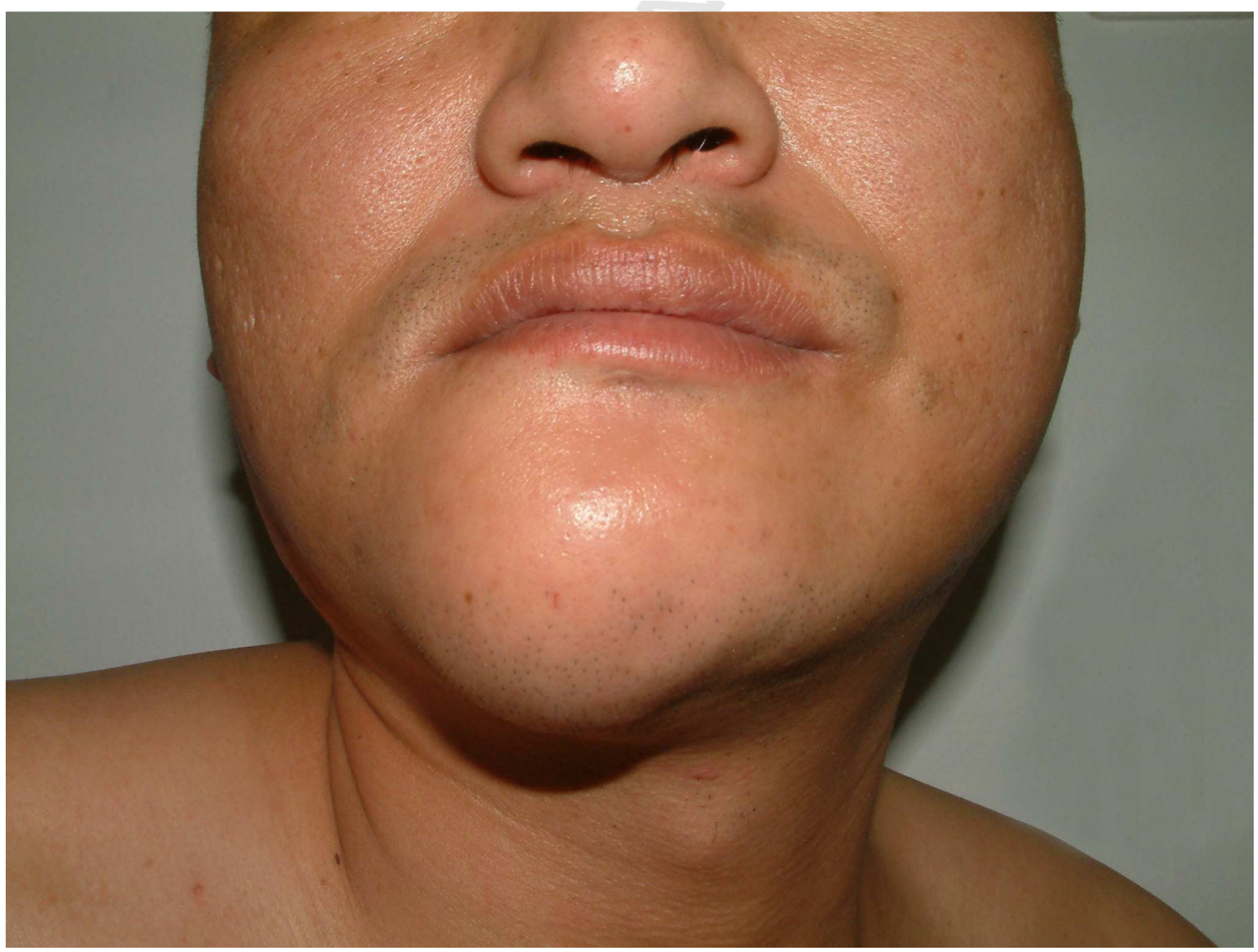

Figure 1.

Clinical photograph of a $39-y e a r-o l d ~ m a n$ with a $4 \times 5 \mathrm{~cm}$ swelling on the mental region. 


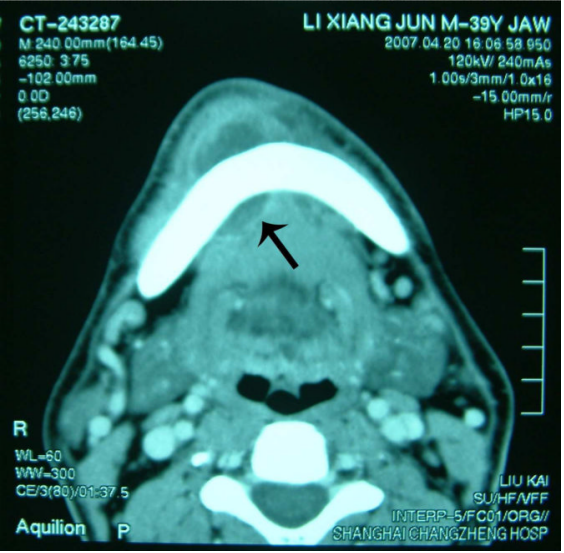

Figure 2.

This axial contrast-enhanced (soft tissue window) CT image shows a well-defined, radiolucent, with peripheral contrast enhancement mass, encircling the cortex of the bone partially (arrow). There is no signs of cortical destruction or medullary involvement. 


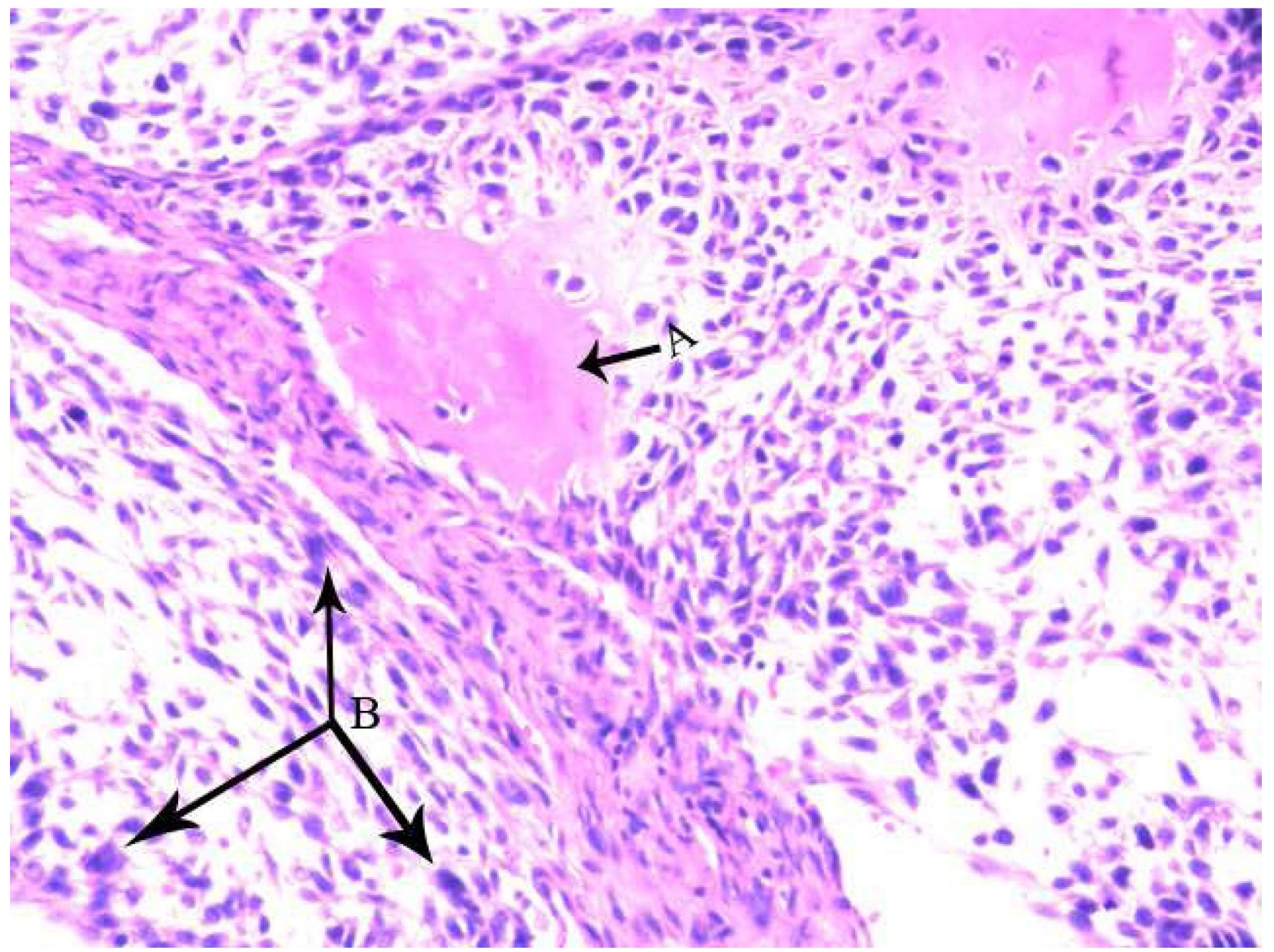




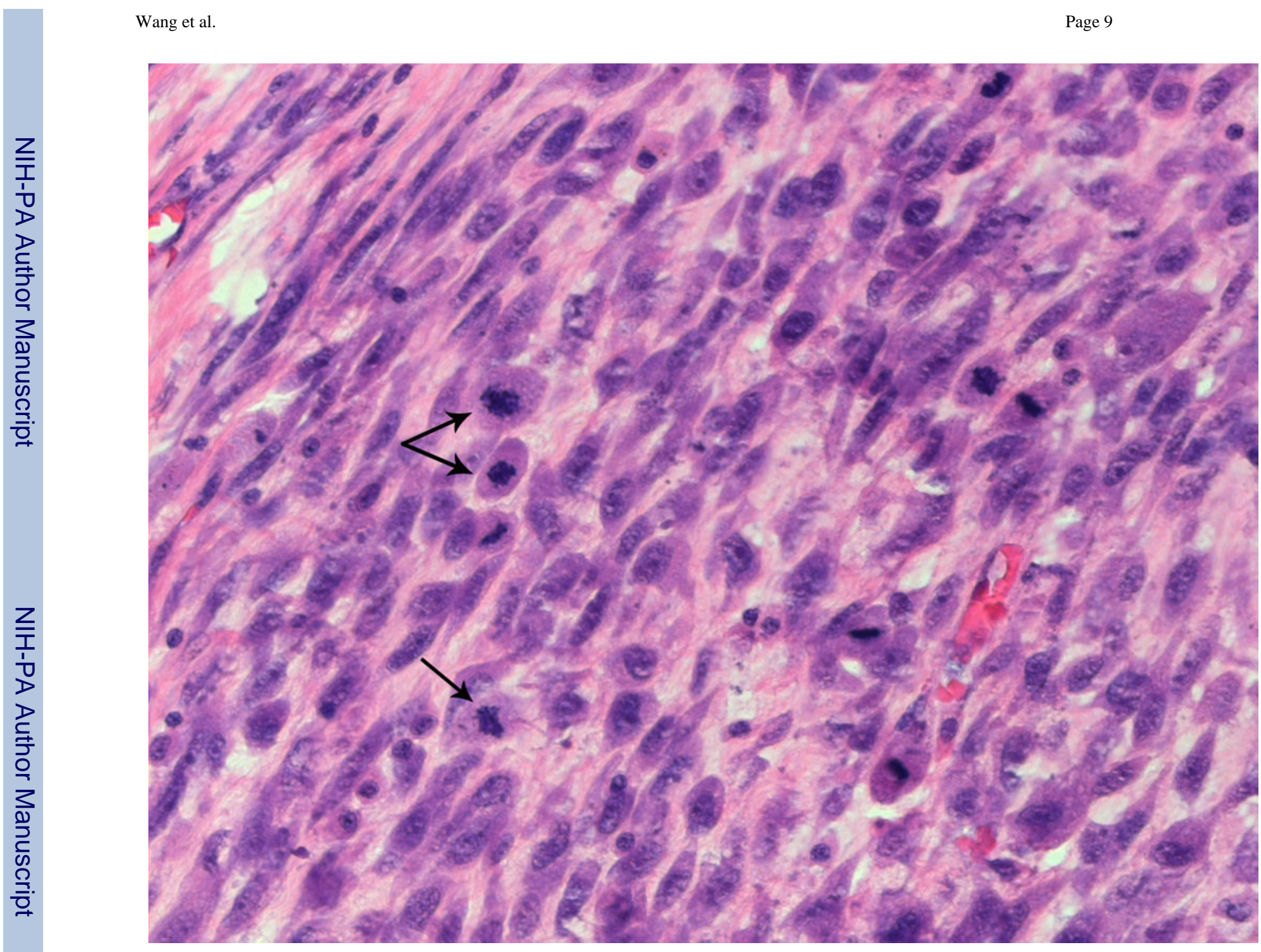

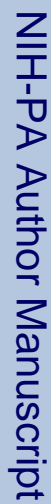

J Oral Maxillofac Surg. Author manuscript; available in PMC 2012 June 1. 


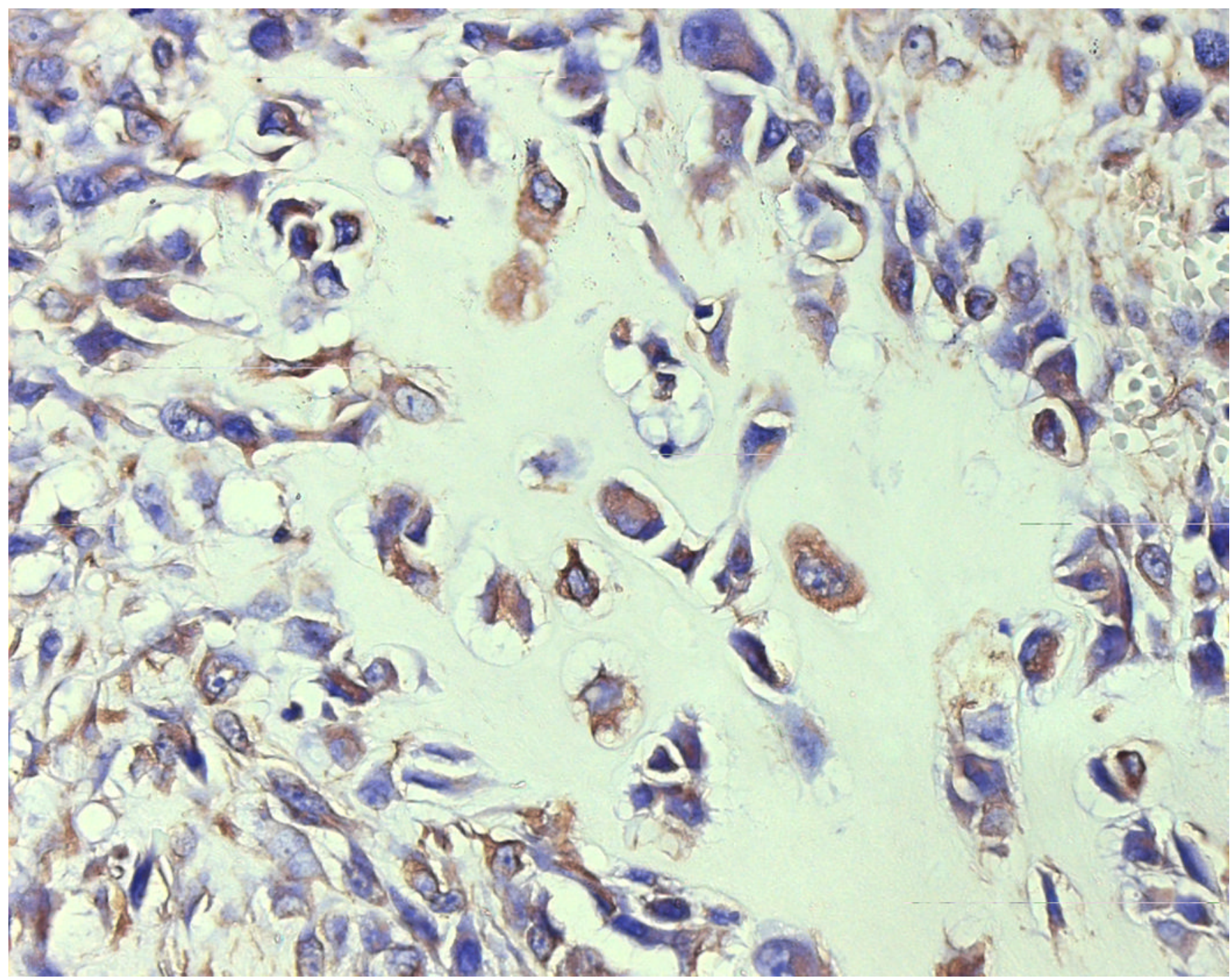

Figure 3.

A. Photomicrograph showing lobules of atypical chondroid admixed with small foci of malignant osteoid formation (arrow A). The tumor consists of highly atypical malignant chondroblastic cells (arrow B). (H\&E, original magnification $\times 100)$.

B. Some mitotic figures are present among the chondrocytes (arrows). (H\&E, original magnification $\times 100$ ).

C. Some tumor cells show positive staining for antibodies against the S-100 protein. (original magnification $\times 100$ ). 

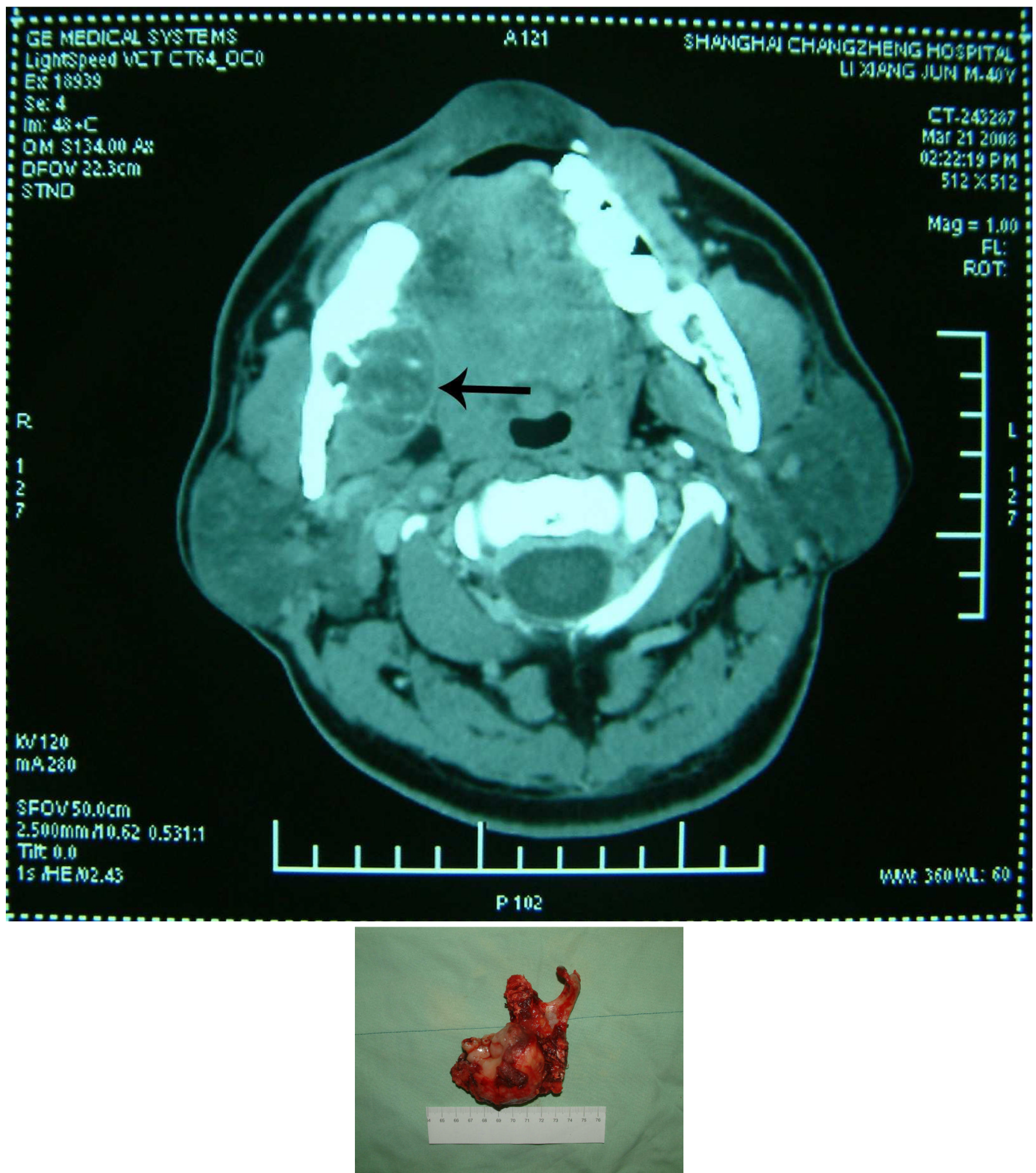

Figure 4.

A. Axial contrast-enhanced (soft tissue window) CT scan demonstrating a recurrent lesion (arrow) on the outer surface of the lingual cortex of the right posterior mandible. 
B. Photograph of the resected recurrent tumor together with the residue of the right mandible. 
Table 1

The key differences in four types of osteosarcomas

\begin{tabular}{|c|c|c|c|c|}
\hline Characteristic & Intramedullary OS & High grade surface OS & Periosteal OS & Parosteal OS \\
\hline \multicolumn{5}{|l|}{ Clinic } \\
\hline Chief complaint & Swelling, pain & Swelling, pain & Swelling & Swelling \\
\hline Progress & Rapid & Rapid & Slow & Slow \\
\hline Prognosis & Poor & Poor & Relatively good & Good \\
\hline \multicolumn{5}{|l|}{ Radiologic } \\
\hline Appearance & $\begin{array}{l}\text { Sclerotic, lytic or } \\
\text { mixed }\end{array}$ & $\begin{array}{l}\text { Fluffy, immature } \\
\text { ossification }\end{array}$ & Radiolucent mass & Radiodense mass \\
\hline Origination & Medullary & Juxtacortical & Juxtacortical & Juxtacortical \\
\hline $\begin{array}{l}\text { Medullary } \\
\text { involvement }\end{array}$ & Yes & None or minimum & None & None \\
\hline \multicolumn{5}{|l|}{ Histologic } \\
\hline Main component & $\begin{array}{l}\text { Tumor osteoid, } \\
\text { osteoblastic, } \\
\text { chondroblastic, or } \\
\text { fibroblastic } \\
\text { stroma. }\end{array}$ & $\begin{array}{l}\text { Identical to } \\
\text { intramedullary OS. } \\
\text { predominantly } \\
\text { osteoblastic stroma }\end{array}$ & $\begin{array}{l}\text { Cartilaginous } \\
\text { tissue, } \\
\text { chondroblastic } \\
\text { stroma }\end{array}$ & $\begin{array}{l}\text { Bone tissue, } \\
\text { fibroblastic stroma }\end{array}$ \\
\hline Grade & High & High & Intermediate & Low \\
\hline Growth pattern & Highly aggressive & Highly aggressive & $\begin{array}{l}\text { Expansile, } \\
\text { locally } \\
\text { aggressive }\end{array}$ & $\begin{array}{l}\text { Expansile, locally } \\
\text { aggressive }\end{array}$ \\
\hline
\end{tabular}

